\section{The association of vitamin D deficiency with tear break-up time and Schirmer testing in non-Sjögren dry eye}

BE Kurtul ${ }^{1}$, PA Özer ${ }^{1}$ and MS Aydinli ${ }^{2}$

\begin{abstract}
Purpose To investigate the effect of vitamin D deficiency on tear break-up time (TBUT) and Schirmer test scores and to assess their relationship in non-Sjögren dry-eye patients. Methods Thirty-four patients with serum vitamin $\mathrm{D}$ deficiency and 21 control subjects with normal vitamin $D$ levels were included in this study. The TBUT and Schirmer-1 test without topical anesthesia were performed to all patients.

Results The mean TBUT were $5.18 \pm 2.15$ and $7.36 \pm 3.10 \mathrm{~s}$ and Schirmer scores were $12.18 \pm 6.44$ and $18.57 \pm 8.99 \mathrm{~mm}$ in the study and control groups, respectively. TBUT scores and Schirmer-1 results of the study group were significantly lower than the control group $(P=0.01$ and 0.007 , respectively). The mean vitamin $D$ levels were $11.50 \pm 1.8 \mathrm{ng} / \mathrm{ml}$ in the study group and $32.8 \pm 8.72 \mathrm{ng} / \mathrm{ml}$ in control group $(P=0.001)$. Dry-eye symptoms were detected in all patients in the study group and $15 \%$ of the patients in the control group.

Conclusions We demonstrated that vitamin D deficiency decreases the TBUT and Schirmer test values and may be associated with dry-eye symptoms in non-Sjögren syndrome.

Eye (2015) 29, 1081-1084; doi:10.1038/eye.2015.96; published online 12 June 2015
\end{abstract}

\section{Introduction}

Vitamin D is a fat-soluble prohormone and has immunomodulatory properties. ${ }^{1-3}$ Vitamin D deficiency has an important role in the development of cancer and several autoimmune diseases. ${ }^{1,2}$ It has also been found that environmental and hormonal factors, the fatsoluble vitamins such as vitamin $\mathrm{D}$, may have a role in the pathogenic immunoregulatory process of primary Sjögren's syndrome (Pss). ${ }^{4-6}$
The vitamin D supplementation is suggested for chronic autoimmune diseases. ${ }^{4}$ Low levels of vitamin D may be related with low-complement components and the presence of cryoglobulins in predicting eventual development of lymphoma in patients with Ss. ${ }^{5}$

Dry eye is a multifactorial condition of the tears and ocular surface that results in symptoms of discomfort, visual disturbance, and tear film instability with potential damage to the ocular surface. ${ }^{5-10}$ It is described by increased osmolarity of the tear film and inflammation of the ocular surface. ${ }^{8}$

It is observed in $\sim 33 \%$ of the world population. Dry-eye disease contains two etiopathogenic groups. These are aqueous teardeficient dry eye and evaporative dry eye. The aqueous tear-deficient group includes Ss and non-Ss dry-eye groups. ${ }^{5}$ Non-Ss dry-eye disease has several causes as lack of lacrimal gland, impairment or dysfunction of the lacrimal gland, reflex block, and drug action. ${ }^{5}$ Non-Ss dry eye can also be secondary to different conditions. ${ }^{5}$ Non-Sjögren dry eye is a form of aqueous tear deficiency when the systemic autoimmune features have been excluded. ${ }^{5}$

Because of close relationship between vitamin $\mathrm{D}$ and inflammation, we aimed to investigate the effect of vitamin D deficiency on tear break-up time (TBUT) and Schirmer test scores in non-Ss patients and to assess their relationship.

\section{Materials and methods}

The study was undertaken between March 2013 and July 2013, in Training and Research Hospital, Department of Ophthalmology and was designed as a prospective clinical study. This submission has received Institutional Review Board/Ethics Committee approval from
${ }^{1}$ Department of Ophthalmology, Dr Sami Ulus Maternity and Children's Health and Diseases Training and Research Hospital, Ankara, Turkey

${ }^{2}$ Department of Family Medicine, Dr Sami Ulus Maternity and Children's Health and Diseases Training and Research Hospital, Ankara, Turkey

Correspondence: BE Kurtul, Department of Ophthalmology, Dr Sami Ulus Maternity and Children's Health and Diseases Training and Research Hospital, Ankara 06080 Turkey Tel: +90 3123056638 ; Fax: +90 3123056649 . E-mail: becekurtul@ yahoo.com

Received: 7 May 2014 Accepted: 13 August 2014 Published online: 12 June 2015 
our institution. Informed consent was obtained from each subject before the study. Described research adhered to the tenets of the declaration of Helsinki. Thirty-four patients with serum vitamin D deficiency and 21 control subjects without serum vitamin D deficiency were included in the study. The patients with Pss or other systemic rheumatic disease history, vitamin B12 deficiency, and ocular surface disorders and wearing contact lenses were excluded from the study. The study patients were referred to our clinic from the family medicine department after the determination of vitamin $\mathrm{D}$ deficiency for a possible dry-eye disease, as these patients complained of dry-eye symptoms. Vitamin D levels were measured for symptoms such as chronic fatigue as well as low sun exposure in the family medicine department. Vitamin D status may be affected by factors such as food fortification and sun exposure. ${ }^{3}$ We asked the patients with an interview about the daily intake of the fatty fish (salmon, tuna, mackerel, and so on), dairy products, egg yolks, and which contain vitamin D. ${ }^{3}$ The nutritional status and eating habits of the patients were similar. Serum concentrations of vitamin D were measured by LCMS/MS method (Shimadzu API 3200, Applied Biosystem, Waltham, MA, USA). ${ }^{11}$ All blood samples were only taken between March and July because of the possible variation throughout the year. The serum vitamin $\mathrm{D}$ levels were described as follows: severe deficiency $<10 \mu \mathrm{g} / \mathrm{l}$, moderate deficiency 10-24 $\mu \mathrm{g} / \mathrm{l}$, desired $>30 \mu \mathrm{g} / \mathrm{l}$, and toxic $>150 \mu \mathrm{g} / 1 .{ }^{11}$ Dry-eye symptoms such as irritation, a foreign body sensation, burning, presence of stringy mucus discharge, and transient blurring of vision were assessed by personal communication with each subject. The symptoms were assessed by the ocular surface disease index (OSDI; Allergan, Irvine, CA, USA) questionnaire. ${ }^{12}$ The OSDI consists of 12 questions on symptoms within the past week and yields scores ranging from 0 (least severe) to 100 (most severe). A score of 12 is typically used as a cutoff for normal, 13-22 for mild dry eye, 23-32 for moderate dry eye, and $\geq 33$ for severe dry eye. ${ }^{13-15}$ The total OSDI score was measured as total OSDI score $\times 25$ / total number of questions answered. ${ }^{13,14}$

The TBUT and Schirmer-1 test were evaluated after a full ophthalmologic examination. TBUT and Schirmer-1 test without topical anesthesia were performed on all patients with 30-min interval after the ophthalmologic examination by the same examiner. Patients were avoided from ocular manipulations before the tests since this may affect the results.

The TBUT was measured after fluorescein staining. Subjects were instructed to blink, and the tear film was examined using the cobalt blue filter of a slit-lamp biomicroscope. The time interval in seconds between the instillment of fluorescein and the appearance of the first randomly distributed dry spot was considered as the TBUT. This method was repeated three times for each eye, and the average of the results was registered as the mean TBUT. ${ }^{12}$ TBUT of $<10 \mathrm{~s}$ was accepted as abnormal.

The Schirmer-1 test was performed without anesthesia by placing a standardized strip of filter paper in the one-third lateral tarsal conjunctiva away from the cornea. Outcomes were expressed in millimeters after $5 \mathrm{~min}$ of wetting. ${ }^{12}$

\section{Statistical analysis}

Data were analyzed using the SPSS 18.0 (Chicago, IL, USA) for Windows. Continuous variables were expressed as mean $\pm \mathrm{SD}$ and categorical variables as percentages. Post hoc test and Mann-Whitney $U$-test was used for statistical evaluations. A two-tailed $P$-value $<0.05$ was regarded as significant.

\section{Results}

The mean age of study group subjects and control subjects were $51 \pm 16$ years (range 28-77years) and $47 \pm 9$ years (range $24-63$ years), respectively $(P=0.2)$. Thirty of them were female and 4 of them were male in the study group, 18 of them were female and 3 of them were male in the control group (Table 1). Dry-eye symptoms such as irritation, a foreign body sensation, burning, and transient blurring of vision were detected in all patients in the study group and $15 \%$ of the patients in the control group. The OSDI scores were 27.77 (18.75-37.50) in and 8.33 (6.25-28.41) in the study and control groups, respectively. The difference between groups was not significant $(P=0.071)$. The mean vitamin $\mathrm{D}$ levels were $11.50 \pm 1.8 \mathrm{ng} / \mathrm{ml}$ in the study group and $32.8 \pm 8.72 \mathrm{ng} /$ $\mathrm{ml}$ in the control group $(P=0.001)$. The mean TBUT and Schirmer-1 scores were $5.18 \pm 2.15 \mathrm{~s}$ and $12.18 \pm 6.44 \mathrm{~mm}$ and $7.36 \pm 3.10 \mathrm{~s}$ and $18.57 \pm 8.99$ mmfor the study control groups, respectively (Table 2 ). The TBUT scores and Schirmer-1 test values of study group were significantly lower than that of the control group $(P=0.01$ and 0.007 , respectively) (Table 2 ).

\section{Discussion}

Clinically, the reduced intake of vitamin D is found to increase the prevalence of certain autoimmune diseases, such as type I diabetes mellitus, multiple sclerosis,

Table 1 The mean age and gender of subjects

\begin{tabular}{lccc}
\hline & $\begin{array}{c}\text { Study group } \\
(\mathrm{n}=34)\end{array}$ & $\begin{array}{c}\text { Control group } \\
(\mathrm{n}=21)\end{array}$ & P-value \\
\hline Mean age \pm SD, years & $51 \pm 16$ years (28-77) & $47 \pm 9$ years (24-63) & 0.2 \\
Gender (female/male) & $30 / 4$ & $18 / 3$ & 0.5 \\
\hline
\end{tabular}


Table 2 The mean vitamin D levels, TBUT, and Schirmer test scores of subjects

\begin{tabular}{lccc}
\hline & Study group $(\mathrm{n}=34)$ & Control group $(\mathrm{n}=21)$ & P-value \\
\hline Serum vitamin D levels, $\mathrm{ng} / \mathrm{ml}($ mean $\pm \mathrm{SD})$ & $11.50 \pm 1.8$ & $32.86 \pm 8.72$ & 0.001 \\
TBUT, seconds (mean $\pm \mathrm{SD})$ & $5.18 \pm 2.15$ & $7.36 \pm 3.10$ & 0.01 \\
Schirmer test scores, $\mathrm{mm}($ mean $\pm \mathrm{SD})$ & $12.18 \pm 6.44$ & $18.57 \pm 8.99$ & 0.007 \\
\hline
\end{tabular}

rheumatoid arthritis, systemic lupus erythematosus, and inflammatory bowel diseases. ${ }^{10}$ Vitamin D-binding protein (DBP) binds the vitamin D metabolites with high affinity. ${ }^{10}$ DBP may have a role as a neutral acute-phase reactant.

The important immunoregulatory functions of the fatsoluble vitamins (vitamins A, D, and E) and their role in the regulation of autoimmune diseases have been shown. ${ }^{16,17}$ Especially, the vitamin D deficiency occurs at a higher rate in patients with autoimmune disorders. ${ }^{11,18,19}$ The vitamin D supplementation is recommended in the treatment of the many rheumatic conditions. ${ }^{17}$

The effect of environmental and hormonal factors, such as vitamin $\mathrm{D}$, was mentioned in the pathogenic process of dry-eye disease. ${ }^{4,6}$ It is now accepted that dry eye is a localized autoimmune disease, and irregular protective immunoregulation and proinflammatory pathways of the ocular surface cause this disorder. ${ }^{20}$

Recent studies have focused on the inflammatory biologic markers in establishing treatment strategies to cover the multifactorial nature of non-Ss dry eye. ${ }^{20}$ Increased levels of proinflammatory cytokines and markers have been observed in the tears and the ocular surface of non-Ss dry-eye patients. Upregulation of inflammatory cytokines, including interleukin- $1 \alpha$ and IL1 $\beta$, has been observed in non-Ss dry eye. ${ }^{20}$

Bang et $a l^{21}$ emphasized the clinical and pathogenic significance of vitamin D metabolites in Pss. Müller et $a l^{22}$ found severely diminished blood concentrations of vitamin D levels in patients with Pss. Agmon-Levin et al ${ }^{4}$ emphasized that low levels of vitamin D correlated with the presence of peripheral neuropathy and lymphoma among Pss patients in their study. A small but favorable effect of higher vitamin D levels on DES symptoms was mentioned in another study. ${ }^{23}$

Owing to the antiinflammatory and immunoregulatory properties of vitamin D, its deficiency may cause dryness of the eyes, which is an autoimmune and inflammatory condition. The relation between vitamin D deficiency and Ss was shown in previous studies. ${ }^{1-4}$

In our study, none of the patients had autoimmune disease such as Ss, which could affect the TBUT and Schirmer levels. We researched the possible link between non-Ss dry eye and vitamin D deficiency. TBUT scores and Schirmer-1 test values of the study group were significantly lower than that of the control group.
Dry-eye symptoms were detected in all patients with diminished serum vitamin D levels. In contrast, the difference in OSDI scores was not significant between groups. This may be due to the small size of the patients in our study. We demonstrated that vitamin D deficiency may be associated with the dry-eye symptoms and lower TBUT and Schirmer values.

Kinds of systemic therapies are being used to induce tear components or mucin secretion and to reduce inflammation and dry-eye symptoms. Some of them are forms of steroidal and nonsteroidal anti-inflammatory agents, oral cholinergic agonists, vitamins A and D, neurotransmitters, and neuropeptides besides usual topical drops used for dry-eye treatment. ${ }^{10}$ The vitamin D supplementation is suggested for chronic autoimmune diseases and recommended in the treatment of many rheumatic conditions. ${ }^{4,13}$ Tincani et al ${ }^{5}$ proposed vitamin D supplementation as an additional mode of optimization of Ss treatment. Because of the associations between hypovitaminosis D and severe complications of Ss, it can be proposed that vitamin $\mathrm{D}$ supplementation should be given to patient with Ss. Although it is difficult to suggest that oral vitamin D supplementation decreases the symptoms of dry eye, as we learned from phone visits, correction of decreased serum vitamin D levels with oral supplementations may reduce the dry-eye symptoms of these patients. As there are no statistically proved data about this subject, it should be the subject of another study.

Hence, evaluation of serum vitamin D levels in patients with dry-eye symptoms is important and should not be disregarded in their clinical follow-up. The role of vitamin D should be further investigated in non-Ss dryeye disease. Further studies with more patients are needed to compare the changes in TBUT and Schirmer scores after the oral supplementation of vitamin $\mathrm{D}$.

\section{Summary}

\section{What was known before}

- The role of the vitamin D deficiency in non-Sjögren dry-eye patients is unclear.

\section{What this study adds}

- Because of the possible link between non-Ss dry eye and vitamin $\mathrm{D}$ deficiency based on inflammation, the evaluation of serum vitamin D levels in patients with dry-eye symptoms is important. 


\section{Conflict of interest}

The authors declare no conflict of interest.

\section{Disclaimer}

The authors have no proprietary interest in any of the materials used in this study. The authors alone are responsible for the content and writing of the paper.

\section{References}

1 Smyk DS, Orfanidou T, Invernizzi P, Bogdanos DP, Lenzi M. Vitamin D in autoimmune liver disease. Clin Res Hepatol Gastroenterol 2013; 37: 535-545.

2 Prietl B, Treiber G, Pieber TR, Amrein K. Vitamin D and immune function. Nutrients 2013; 5(7): 2502-2521.

3 Health Quality Ontario. Clinical utility of vitamin d testing: an evidence-based analysis. Ont Health Technol Assess Ser 2010; 10(2): 1-93.

4 Agmon-Levin N, Kivity S, Tzioufas AG, Lopez Hoyos M, Rozman B, Efes I et al. Low levels of vitamin-D are associated with neuropathy and lymphoma among patients with Sjögren's syndrome. J Autoimmun 2012; 39(3): 234-239.

5 Tincani A, Andreoli L, Cavazzana I, Doria A, Faverom M, Fenini MG et al. Novel aspects of Sjögren's syndrome in 2012. BMC Med 2013; 11: 93 .

6 Stevenson W, Chauhan SK, Dana R. Dry eye disease: an immune-mediated ocular surface disorder. Arch Ophthalmol 2012; 130(1): 90-100.

7 Labb'e A, Liang Q, Wang Z, Zhang Y, Xu L, Baudouinet C et al. Corneal nerve structure and function in patients with nonSjögren dry eye: clinical correlations. Invest Ophthalmol Vis Sci 2013; 54: 5144-5150.

8 The definition and classification of dry eye disease: report of the definition and classification subcommittee of the International Dry Eye WorkShop (2007). Ocul Surf 2007; 5(2): 75-92.

9 Peral A, Dominguez-Godinez CO, Carracedo G, Pintor J. Therapeutic targets in dry eye syndrome. Drug News Perspect 2008; 21(3): 166-176.

10 Sahillioğlu B, Muhittin A, Serdar N, Erkal, Erden G, Bakır F et al. Method validation of tandem mass spectrometry for 25-hydroxyvitamin D3 and comparison of this method with other methods. Turk J Biochem 2011; 36(1): 73-79.
11 Alves M, Reinach PS, Paula JS, Vellasco E, Cruz AA Bachette $\mathrm{L}$ et al. Comparison of diagnostic tests in distinct well-defined conditions related to dry eye disease. PLoS One 2014; 9(5): e97921.

12 Miller KL, Walt JG, Mink DR, Satram-Hoang S, Wilson SE, Perry HD et al. Minimal clinically important difference for the ocular surface disease index. Arch Ophthalmol 2010; 128(1): 94-101.

13 Schiffman RM, Christianson MD, Jacobsen G, Hirsch JD, Reis BL. Reliability and validity of the ocular surface disease index. Arch Ophthalmol 2000; 118(5): 615-621.

14 Tung CI, Perin AF, Gumus K, Pflugfelder SC. Tear meniscus dimensions in tear dysfunction and their correlation with clinical parameters. Am J Ophthalmol 2014; 157(2): 301-310.

15 Demir HD, Aydın E, Sezer E, Yardım H. Evaluation of plasma vitamin A and E levels and tear film changes in patients with Psoriasis Vulgaris. Korean J Ophthalmol 2013; 27(3): 158-161.

16 Szodoray P, Horvath IF, Papp G, Barath S, Gyimesi E, Csathy $\mathrm{L}$ et al. The immunoregulatory role of vitamins $\mathrm{A}$, $\mathrm{D}$ and $\mathrm{E}$ in patients with primary Sjögren's syndrome. Rheumatology 2010; 49(2): 211-217.

17 Abrahamsen B, Harvey NC. The role of vitamin D supplementation in patients with rheumatic diseases. Nat Rev Rheumatol 2013; 9(7): 411-422.

18 Pelajo CF, Lopez-Benitez JM, Miller LC. Vitamin D and autoimmune rheumatologic disorders. Autoimmun Rev 2010; 9(7): 507-510.

19 Stern ME, Schaumburg CS, Pflugfelder SC. Dry eye as a mucosal autoimmune disease. Int Rev Immunol 2013; 32(1): 19-41.

20 Na KS, Mok JW, Kim JY, Joo CK. Proinflammatory gene polymorphisms are potentially associated with Korean non-Sjogren dry eye patients. Molecular Vision 2011; 17: 2818-2823.

21 Bang B, Asmussen K, Sorensen OH, Oxholm P. Reduced 25-hydroxyvitamin D levels in primary Sjögren's syndrome. Correlations to disease manifestations. Scand J Rheumatol 1999; 28(3): 180-183.

22 Müller $\mathrm{K}$, Oxholm $\mathrm{P}$, Sørensen $\mathrm{OH}$, Sorensen $\mathrm{OH}$, Thymann M, Hoier-Madsen M et al. Abnormal vitamin D3 metabolism in patients with primary Sjogren's syndrome. Ann Rheum Dis 1990; 49: 682-684.

23 Galor A, Gardener H, Pouyeh B, Feuer W, Florez H. Effect of a Mediterranean dietary pattern and vitamin $D$ levels on dry eye syndrome. Cornea 2014; 33(5): 437-441. 